\section{Effects of multipath propagation delay on uplink performance of synchronous DS-CDMA systems communicating in dispersive Rayleigh fading channels}

\section{S.-H. Hwang and L. Hanzo}

\begin{abstract}
The effect of the maximum asynchronous arrival time difference (AATD) of the delayed multipath components on the uplink performance of synchronous DS-CDMA systems is investigated, when communicating in a frequency-selective Rayleigh fading channel. Numerical results demonstrate that the achievable user capacity gain may be as high as $64 \%$ at a bit error rate of $10^{-2}$, when the maximum AATD is sufficiently low.
\end{abstract}

Introduction: Synchronous DS-CDMA uplink (UL) transmission has been proposed for reducing the effects of multiple access interference (MAI) [1], with the additional benefit of having a lower multi-user detection or interference cancellation complexity than asynchronous systems [2]. Synchronous UL DS-CDMA is therefore considered an attractive technique for employment in future mobile communication systems [3] or in mobile broadband wireless access [4], provided that sufficiently accurate adaptive timing advance control can be invoked for adjusting the arrival time of the first RAKE receiver branch to render it quasi-synchronous. It is plausible that the main paths of all asynchronous users may be synchronised, but the delayed multipath components cannot be synchronised. Hence, previous studies [1, 5] have assumed the remaining delayed RAKE receiver branches to be asynchronous and have neglected the dependence between the arrival time of the synchronous main path and the delayed asynchronous multipath components. In reality, the arrival time of the asynchronous delayed multipath components may exhibit a non-negligible dependence on or correlation with that of the synchronous main UL path, although to the best of our knowledge the impact of this path arrival-time correlation has not been studied in the literature. Against this background, the novel contribution of this Letter is that of considering the performance of the synchronous DS-CDMA UL as well as quantifying the interrelationship of the maximum asynchronous arrival time difference (AATD) of the delayed multipath components and the spreading code orthogonality. More explicitly, using the expressions derived, both the bit error rate (BER) and the achievable user capacity are evaluated and compared to that of conventional asynchronous CDMA, where the main path is not synchronised either.

System model and analysis: The synchronous DS-CDMA UL described in [1] is considered, where orthogonal spreading sequences are used. Let us assume that we have $K$ transmitters that are capable of adjusting their timing instants with respect to the main paths to be aligned with that of the base station using adaptive timing advance control [1, 3], where the arrival time of the first RAKE receiver branch signal is assumed to be synchronous, while the remaining branch signals are asynchronous within a maximum AATD interval limited to a fraction of one symbol duration. Assuming dispersive Rayleigh fading, the negative-exponentially decaying multipath intensity profile (MIP) expressed as $E\left[\left\{\beta_{l}^{(k)}\right\}^{2}\right]=\Omega_{0}^{(k)} e^{-l \delta}, \delta \geq 0$ is considered, where $\beta_{l}^{(k)}$ refers to the Rayleigh distributed envelope of the $l$ th faded path of the $k$ th user, $\Omega_{0}^{(k)}$ is the power associated with the first faded path, and $\delta$ reflects the negative exponential decay factor of the average path power against path delay. A coherent RAKE receiver is used, where the number of taps $L_{r}$ is a variable that is less than or equal to the actual number of paths $L^{(k)}$ encountered by the $k$ th user. The RAKE receiver's tap weights and phases are assumed to be perfect estimates of the channel parameters. Perfect power control is assumed.

For the user of interest $(k=1)$, the variance of the MAI term at the output of the RAKE receiver is given by

$$
\begin{aligned}
\sigma_{\text {mai }, n}^{2}= & \frac{E_{b}}{2 T} \sum_{k=2}^{K} \sum_{l=0}^{L^{(k)}-1}\left\{\beta_{n}^{(1)}\right\}^{2} E\left\{\beta_{l}^{(k)}\right\}^{2} E\left[\left\{b_{-1}^{(k)} R W_{k 1}\left(\tau_{n l}^{(k)}\right)\right.\right. \\
& \left.\left.+b_{0}^{(k)} R \hat{W}_{k 1}\left(\tau_{n l}^{(k)}\right)\right\}^{2}\right] E\left[\cos \left(\varphi_{n l}^{(k)}\right)\right]
\end{aligned}
$$

with $b_{0}^{(1)}$ being the information bit to be detected, while $b_{-1}^{(1)}$ is the preceding bit, $E_{b}$ is the signal energy per bit and $T$ is the data bit duration. Furthermore, we have $\tau_{n l}^{(k)}=\tau_{l}^{(k)}-\tau_{n}^{(1)}$ where $\tau_{l}^{(k)}$ is the $l$ th multipath delay of the $k$ th user, $\varphi_{n l}^{(k)}=\varphi_{l}^{(k)}-\varphi_{n}^{(1)}$ where $\varphi_{l}^{(k)}$ is the phase of the $l$ th path of the $k$ th user, and $R W_{k 1}$ as well as $R \hat{W}_{k 1}$ are the continuous partial cross-correlation functions of the Walsh-PN spreading sequences as defined in [1] (the reason for assuming Walsh-PN spreading is that these codes may be utilised for both channelisation and randomisation). The cross-correlations can be conveniently formulated in terms of their corresponding discrete aperiodic cross-correlations $C W_{k, 1}(i)$ expressed as:

$$
C W_{k, 1}(i)= \begin{cases}\sum_{j=0}^{N-1-i} a_{j} w_{j}^{(k)} a_{j+i} w_{j+i}^{(1)}, & 0<i \leq N-1 \\ \sum_{j=0}^{N-1+i} a_{j-i} w_{j-i}^{(k)} a_{j} w_{j}^{(1)}, & -(N-1) \leq i<0 \\ 0, & \text { otherwise }\end{cases}
$$

where $N$ is the processing gain, $w_{j}^{k}$ represents the sign of the $j$ th chip for the $k$ th user's orthogonal spreading sequence, $a_{j}$ represents the sign of the $j$ th chip for the PN sequence. Furthermore, let $I_{k}$ be the correlator output induced by the $k$ th interferer. Then the variance of $I_{k}$ for $n=0$ in (1) is given by

$$
\begin{aligned}
\operatorname{Var}\left(I_{k}\right)= & \frac{E_{b} T_{c}}{12 N \Delta}\left[\sum_{i=1}^{\Delta} C W_{k, 1}^{2}(1+i-N)+C W_{k, 1} \times(1+i-N)\right. \\
& \times C W_{k, 1}(i-N)+C W_{k, 1}^{2}(i-N)+C W_{k, 1}^{2}(i+1) \\
& \left.+C W_{k, 1}^{2}(i)+C W_{k, 1}(i) \times C W_{k, 1}(i+1)\right]
\end{aligned}
$$

where $\Delta$ is the maximum AATD of the delayed multipath components. The factor $1 / \Delta$ and the summation from $i=1$ to $\Delta(0<\Delta<N-1)$ in (3) arise due to having $\Delta$ chip intervals in the time interval of $\left[T_{c},(\Delta+1)\right.$ $\left.T_{c}\right)$, and $\tau_{0 l}^{(k)}$ may fall into any of them with equal probability. Using Pursely's random chip model for the spreading sequences [6] renders $\operatorname{Var}\left(I_{k}\right)$ a random variable, and upon taking the expectation of (3), we arrive at

$$
\begin{aligned}
E\left[\operatorname{Var}\left(I_{k}\right)\right]= & \frac{E_{b} T}{24 N^{2} \Delta}[(\Delta+1)(\Delta+2)-2+\Delta(\Delta+1) \\
& +(N-2)(N-1)-(N-\Delta-2)(N-\Delta-1) \\
& +N(N-1)-(N-\Delta-1)(N-\Delta)] \\
\equiv & E_{b} T C_{1}
\end{aligned}
$$

Similarly, for the expectation of $\operatorname{Var}\left(I_{k}\right)$ it may be shown that assuming $n \geq 1$ yields

$$
\begin{aligned}
E\left[\operatorname{Var}\left(I_{k}\right)\right]= & \frac{E_{b} T}{24 N^{2}(\Delta+1)}[3(\Delta+1)(\Delta+2)-2+(N-1) \times N \\
& -(N-\Delta-1)(N-\Delta)] \\
\equiv & E_{b} T C_{2}
\end{aligned}
$$

The output of the receiver conditioned on $\beta_{n}^{(1)}$ is a Gaussian random process having a mean given by $U_{s}=\sqrt{ }\left(E_{b} T / 2\right) \sum_{n=0}^{L_{r}-1}\left\{\beta_{n}^{(1)}\right\}^{2}$, while upon exploiting that the MAI variances associated with $n=0$ and $n \geq 1$ are formulated in (4) and (5), respectively, the variance of the total interference is as follows:

$$
\begin{aligned}
\sigma_{T}^{2}= & \left(E_{b} T \Omega_{0}\right)\left[C_{1}(K-1)\left\{q\left(L^{(k)}, \delta\right)-1\right\}\right. \\
& \times \frac{\left\{\beta_{0}^{(1)}\right\}^{2}}{\left\{\beta_{0}^{(1)}\right\}^{2}+\sum_{n=1}^{L_{r}-1}\left\{\beta_{n}^{(1)}\right\}^{2}}+C_{2}(K-1) q\left(L^{(k)}, \delta\right) \\
& \left.\times \frac{\sum_{n=1}^{L_{r}-1}\left\{\beta_{n}^{(1)}\right\}^{2}}{\left\{\beta_{0}^{(1)}\right\}^{2}+\sum_{n=1}^{L_{r}-1}\left\{\beta_{n}^{(1)}\right\}^{2}}+\frac{\eta_{0}}{4 E_{b} \Omega_{0}}\right] \times \sum_{n=0}^{L_{r}-1}\left\{\beta_{n}^{(1)}\right\}^{2}
\end{aligned}
$$

where we have $q\left(L^{(k)}, \delta\right)=\sum_{l=0}^{L^{(k)}-1} e^{-l \delta}=\left(1-e^{-L^{(k)} \delta}\right) /\left(1-e^{-\delta}\right)$. The effect of interchip interference was ignored here for the sake of simplicity, since the analysis in [7] shows that the MAI term will dominate the total noise variance, when the number of users is sufficiently high. The variance of the additive white Gaussian noise 
was given in [7]. The probability of error, when encountering a negative-exponentially decaying MIP, is then expressed as

$$
P_{e}^{l}=\int_{0}^{\infty} \int_{0}^{\infty} Q\left(\sqrt{\frac{U_{s}^{2}}{\sigma_{T}^{2}}}\right) \frac{1}{\Omega_{0}} e^{-y / \Omega_{0}} \sum_{k=1}^{L_{r}-1} \frac{\pi_{k}}{\Omega_{k}} e^{-x / \Omega_{k}} d x d y
$$

where $Q(x)=(1 / \sqrt{ }(2 \pi)) \int_{x}^{\infty} \exp \left(-u^{2} / 2\right) d u$ and $\pi_{k}=\Pi_{i=1 ; i \neq k}^{L^{r-1}} \quad x_{k} /$ $\left(x_{k}-x_{i}\right)=\prod_{i=1 ; i \neq k}^{L_{r}-1} \Omega_{k} /\left(\Omega_{k}-\Omega_{i}\right)$.

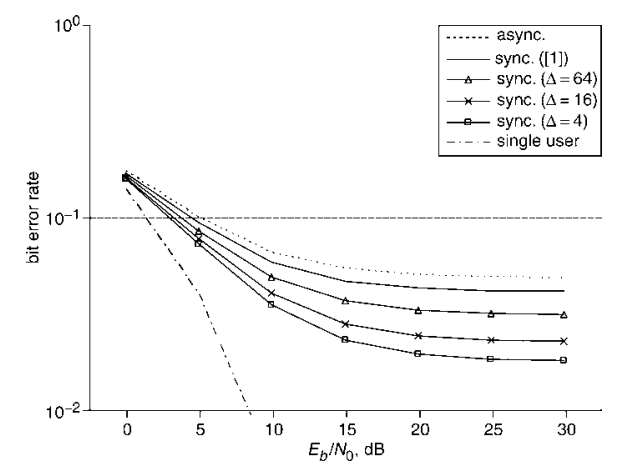

Fig. 1 BER against $E_{b} / N_{0}$ for different values of multipath propagation delay

$\delta=0.2 ;$ number of users 72

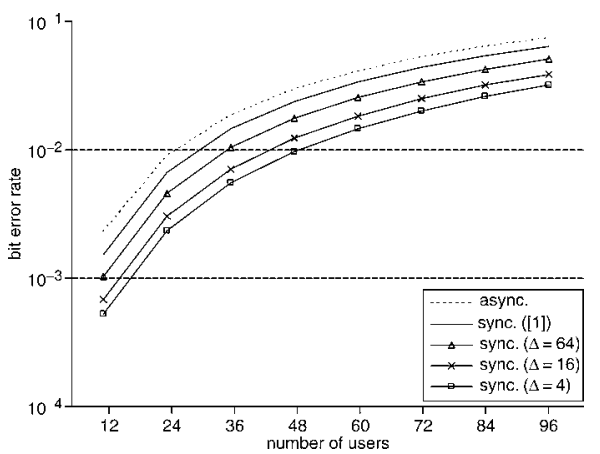

Fig. 2 BER against number of users for different values of multipath propagation delay

$\delta=0.2 ; E_{b} / N_{0}=20 \mathrm{db}$

Results and discussions: Let us now investigate the effects of the specific choice of the maximum AATD of the delayed multipath components $\Delta$ on the achievable UL performance of a coherent BPSK RAKE receiver in a synchronous DS-CDMA system in terms of the average BER and the number of users, when communicating over frequency-selective Rayleigh fading channels. The BER analysis of an asynchronous CDMA system communicating over Rayleigh fading channels can be found in [7]. Fig. 1 shows the achievable average BER performance evaluated from (7) against $E_{b} / N_{0}$, when the MIP's negative-exponential decay factor is assumed to be $\delta=0.2$. We have chosen the parameters of $K=72, N=128, L^{(k)}=5$, and $L_{r}=3$ for characterising the system's performance, but we vary the value of the maximum AATD $\Delta$ of the delayed multipath component, using values of 4,16 and 64 chip duration. The performance of the synchronous UL characterised in [1] is also shown as a benchmark in Fig. 1, when the maximum AATD is set to one data bit duration (128 chips). Fig. 1 shows that the achievable BER performance improves upon decreasing the value of $\Delta$, since the delayed multipath components of the interfering sources arrive within a narrower maximum multipath propagation delay interval and hence the attainable performance of the synchronous UL becomes similar to that of the synchronous downlink. Although to the best of our knowledge it has not been quantified in the literature to what degree the arrival time of the asynchronous multipath propagation components is correlated with that of the main path in the synchronous UL, it is plausible that the impact of the maximum AATD of the delayed multipath components on the achievable UL performance of synchronous DS-CDMA systems must not be ignored. The BER performance of the system quantified against number of users, in conjunction with $E_{b} / N_{0}=20 \mathrm{~dB}, L^{(k)}=5$ and $L_{r}=3$ is plotted in Fig. 2 for different values of $\Delta$. The achievable user capacity shows a $64 \%$ difference between the scenarios when the maximum multipath propagation delay is distributed randomly in a fraction of $1 / 32$ of the symbol duration and that associated with one symbol duration at a bit error rate of $10^{-2}$.

Conclusion: We conclude that the UL capacity of synchronous DSCDMA systems is dependent on the maximum AATD of the delayed multipath components. Deriving an adequate correlation model for the multipath propagation delay constitutes the topic of further research.

(C) IEE 2004

31 August 2004

Electronics Letters online no: 20046802

doi: 10.1049/el:20046802

S.-H. Hwang and L. Hanzo (School of Electronics and Computer Sciences, University of Southampton, Highfield, Southampton SO17 1BJ, United Kingdom)

\section{References}

1 Hong, E.K., et al:: 'Synchronous transmission technique for the reverse link in DS-CDMA terrestrial mobile systems', IEEE Trans. Commun., 1999, 47, (11), pp. 1632-1635

2 Hanzo, L., et al.: 'Single- and multicarrier DS-CDMA' (John Wiley \& IEEE Press, New York, 2003)

3 3GPP TR25.854: Uplink synchronous transmission scheme. (ftp://ftp.3gpp.org)

4 IEEE 802.20 C802.20-03/29: Smart antenna and MC-SCDMA, April 2003

5 Hashem, B.: 'Reverse link capacity of synchronous CDMA systems', Proc. IEEE Vehicular Technology Conf., VTC 2002-Fall, Birmingham, AL, USA, September 2002, pp. 817-820

6 Pursley, M.P.: 'Performance evaluation for phase-coded spread-spectrum multiple-access communication - Part I: System analysis', IEEE Trans. Commun., 1977, 25, (8), pp. 795-799

7 Eng, T., and Milstein, L.B.: 'Coherent DS-CDMA performance in Nakagami multipath fading', IEEE Trans. Commun., 1996, 44, (9), pp. $1117-1129$ 